\title{
Giant Transiting Planets Observations GITPO
}

\author{
C. Afonso ${ }^{1}$, Th. Henning ${ }^{1}$, D. Weldrake ${ }^{1}$, \\ T. Mazeh ${ }^{2}$ and S. Dreizler ${ }^{3}$ \\ ${ }^{1}$ Max-Planck Institut für Astronomie, Heidelberg, Königstuhl 17, 69117 Germany \\ email: afonso@mpia.de \\ ${ }^{2}$ Tel Aviv University, Tel Aviv 69978, Israel \\ email: mazeh@wise.tau.ac.il \\ ${ }^{3}$ Institut für Astrophysik, Universität Göttingen, D-370077 Göttingen, Germany \\ email: dreizler@astro.physik.uni-goettingen.de
}

\begin{abstract}
The search for extrasolar planets is nowadays one of the most promising science drivers in Astronomy. The radial velocity technique proved to be successful in planet hunting, harvesting more than a hundred planets to date. In these last recent years, the transit method has come to fruition, with the detection of seven Jupiter-mass extrasolar transiting planets in close-in orbits $(<0.05 \mathrm{AU})$. Currently, the radius of planets can only be determined from transiting planets, representing the principal motivation and strength of this technique. The MPIA is presently building the Large Area Imager (LAIWO) for the $1 \mathrm{~m}$ telescope in the Wise Observatory, Israel. LAIWO will have a field of view of one square degree. An intensive search for extra-solar planets will be performed with the $1 \mathrm{~m}$ Wise telescope, together with the $1.2 \mathrm{~m}$ MONET telescope in Texas. We will monitor three fields at a given time during three years and more than 200 nights per year. We expect several dozens of extra-solar planets.
\end{abstract}

Keywords. instrumentation: detectors, techniques : image processing, stars: planetary systems

\section{Introduction}

Extrasolar planets searches have gained major importance in the recent years and revealed itself as one of the most promising science drivers in Astronomy. Unexpected results have led to new clues about the formation and evolution of planets. An example is the detection of massive planets, with Jupiter masses, at short periods $P \leqslant 10$ days, indicating that a migration process took place, after acquiring the bulk of their mass. The radial velocity (RV) technique proved to be successful in planet hunting, harvesting more than a hundred planets to date (Mayor\&Queloz (1995); Marcy \& Butler (1996)). Recently, the Earth-mass range was attained with the detection of several Neptune-mass planets with masses equal to 7.5, 14, 18 and $21 M_{\odot}$ (Rivera et al. (2004), Santos et al. (2004), McArthur et al. (2004), Butler et al. (2004)).

Although this technique is undoubtly successful, the degeneracy between the planetary mass and the inclination angle of the orbit, only allows to obtain a minimal mass for the planet. Another promising method has come to fruition in the recent years, with the detection of seven Jupiter-size transiting extrasolar planets in close-in orbits $(<0.05 \mathrm{AU})$ : HD209458b (Charbonneau et al. (2000)), OGLE-TR-56,OGLE-TR-111, OGLE-TR-113, OGLE-TR-132, OGLE-TR-158 (Udalski et al. (2002)) and TrES-1 (Alonso et al. (2004)). Planetary transits, in addition to the mass and the orbital radius, yield the radius of the planet and the inclination angle, provided the light curve has a high photometric accuracy, a high time sampling and the radius of the parent star is known or a stellar mass-radius relation is assumed. Currently, the radius of planets can only be determined 
from transiting planets, representing the principal motivation and strength of the transit technique. A radius measurement is an important quantity, since it allows to constrain the evolutionary and migration history of the planet and to infer its composition and atmosphere through evolutionary models. Furthermore, transit surveys do not pre-select the target stars to be monitored, as radial velocity searches do. In the coming years, several hundred transiting planets are expected to be discovered from the ground, with more than 20 experiments on-going or being planned (see reference for Planets Encyclopedia), as well as from space with missions such as COROT and Kepler (see references).

\section{The Method - Planetary Transits}

The detection method relies on the temporary drop in the brightness of the parent star harboring the planet. If the planetary system is in a favorable orientation relative to the line of sight (nearly perpendicular to the plane of the sky), then once per orbit the planet passes between its star and the observer, causing an occultation or transit that results in a dip in the light curve (see figure 1). Since the fractional change in brightness $\delta \mathrm{F}$ (or transit depth) is proportional to the stellar surface eclipsed by the planetary disk, the planet's size $R_{p}$ can be inferred from photometric measurements, provided the stellar radius is known $\left(\delta \mathrm{F}=\left(\frac{\mathrm{R}_{\mathrm{p}}}{\mathrm{R}_{\star}}\right)^{2}\right)$. For Jupiter-sized planets transiting sun-sized stars, the expected transit depth will be about $1 \%$.

If three or more transits can be measured and confirmed to be due to the same planet, the period $\mathrm{P}$ can be determined and hence from Kepler's third law $\mathrm{P}=\sqrt{4 \pi^{2} \mathrm{a}^{3} / \mathrm{GM}_{\star}}$, the orbital radius or semi-major axis $a$ can also be inferred. Once the radius of the planet and the period are known, the inclination can be determined from the shape of the transit light curve, as parameterized by the ratio of the duration of the transit's flat bottom to

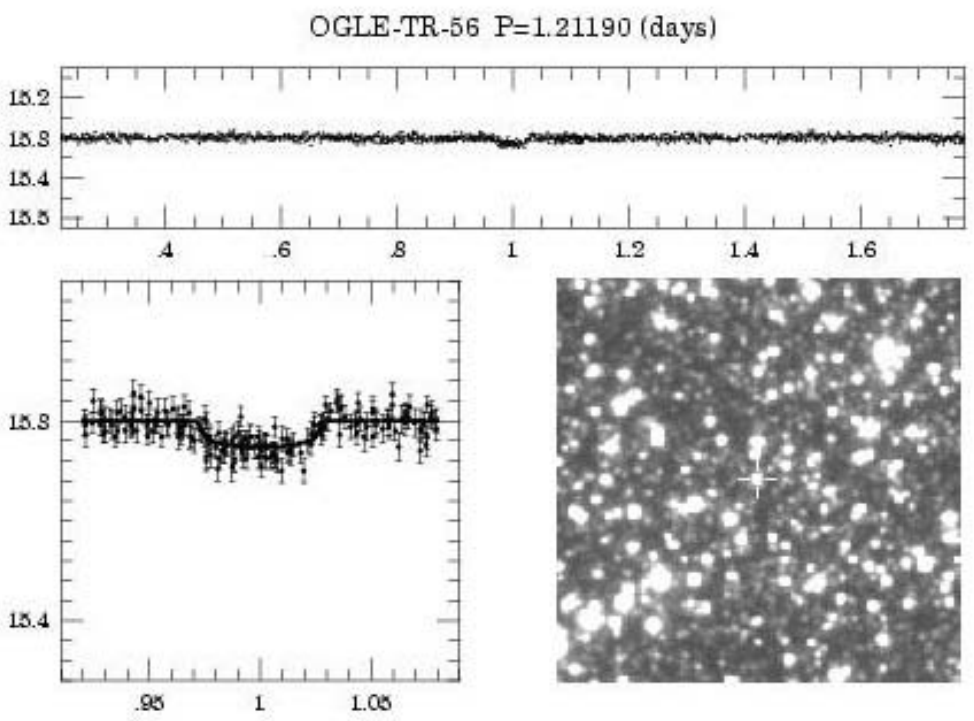

Figure 1. Light curve of OGLE-TR-56, the first planetary system discovered by the transit method. The extra-solar planet has a mass of $0.9 \mathrm{M}_{\mathrm{Jup}}$ and a distance of $0.023 \mathrm{AU}$ to its parent star (Udalski et al. 2002). 
the total transit duration $t_{T}$,

$$
\left(\frac{\mathrm{t}_{\text {flat }}}{\mathrm{t}_{\mathrm{T}}}\right)^{2}=\frac{\left[1-\mathrm{R}_{\mathrm{p}} / \mathrm{R}_{\star}\right]^{2}-\left[\left(\mathrm{D} / \mathrm{R}_{\star}\right) \cos \mathrm{i}\right]^{2}}{\left[1+\mathrm{R}_{\mathrm{p}} / \mathrm{R}_{\star}\right]^{2}-\left[\left(\mathrm{D} / \mathrm{R}_{\star}\right) \cos \mathrm{i}\right]^{2}}
$$

The total transit duration being equal to

$$
\mathrm{t}_{\mathrm{T}}=\frac{\mathrm{PR} \mathrm{R}_{\star}}{\pi \mathrm{a}} \sqrt{\left(1+\frac{\mathrm{R}_{\mathrm{p}}}{\mathrm{R}_{\star}}\right)^{2}-\left(\frac{\mathrm{a}}{\mathrm{R}_{\star}} \operatorname{cosi}\right)^{2}}
$$

\section{The Project - Giant Transiting Planets Observations - GITPO}

The Max-Planck für Astronomie, the University of Tel Aviv and the Sternwarte of Göttingen initiated a transit search program, the Giant Transiting Planets Observations GITPO (see reference), funded by the Max-Planck für Astronomie and the GermanIsraeli Foundation (GIF). The aim of the project is to detect Jupiter-size extra-solar planets around main-sequence stars with magnitudes down to $\mathrm{V}=17$. Currently, a Large Area Imager for the Wise Observatory (LAIWO) is beeing built at the Max-Planck für Astronomie. LAIWO is composed by an array of four front-side illuminated Lockheed CCDs with $4 \mathrm{~K} \times 4 \mathrm{~K}$ pixels, with a pixel size of $15 \mu \mathrm{m}$ (see fig. 2). The camera will be mounted on the $1 \mathrm{~m}$ telescope in the Wise Observatory, in the Negev desert, Israel (see fig. 3). The field of view will be one square degree. The observing strategy will consist in the continuous monitoring of three fields at any given time, until 3,000 image are acquired. We anticipate to have more than 200 nights allocated per year during three years, covering a total sky surface of $30 \mathrm{deg}^{2}$. Observations will be coordinated with the $1.2 \mathrm{~m}$ MONET (see reference) telescope located in Texas, USA, operated by the University

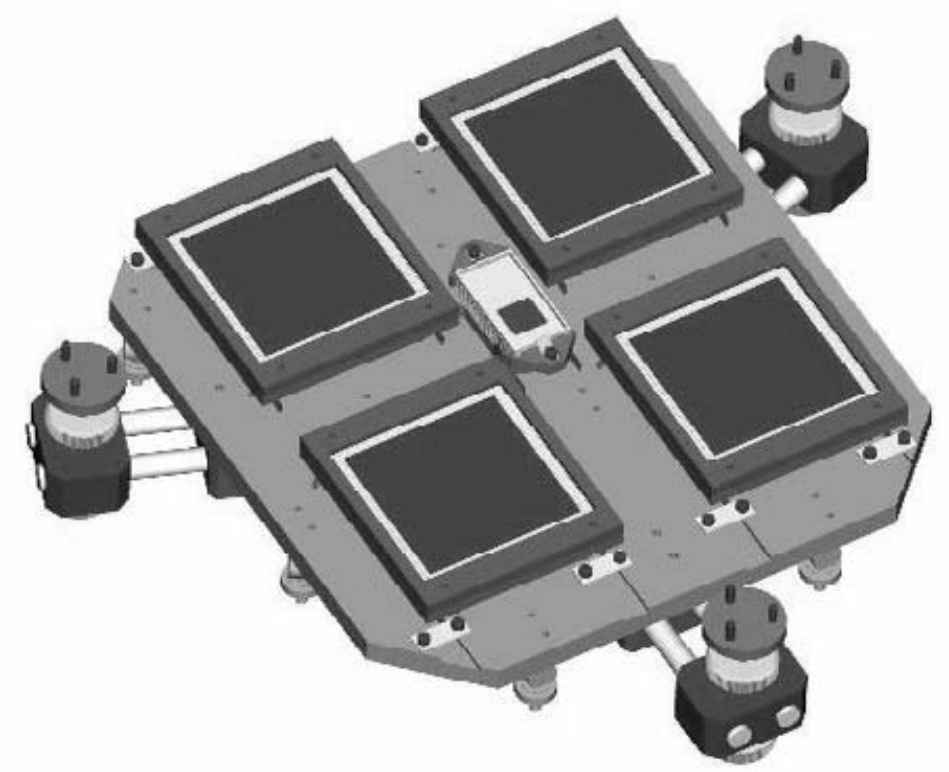

Figure 2. The CCD mosaic of the LAIWO camera. The four CCDs as well as the guider CCD at the center are shown. 


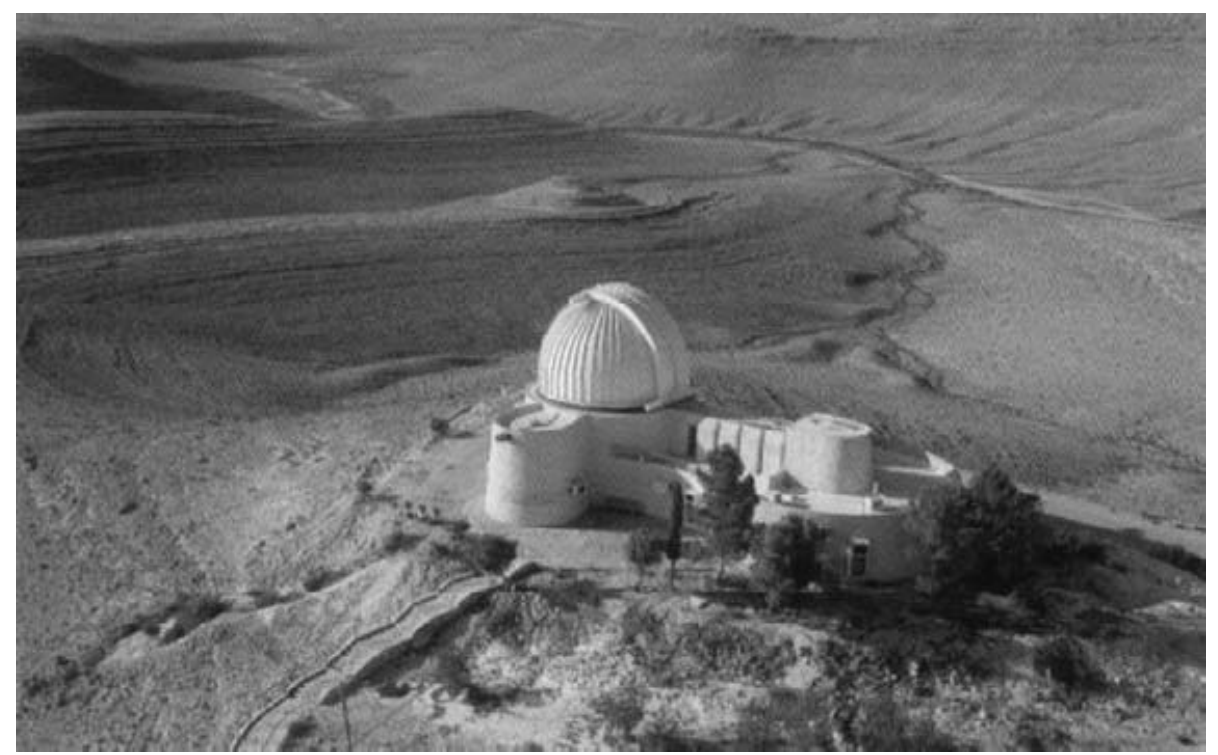

Figure 3. The Wise Observatory in the Negev desert, $200 \mathrm{~km}$ South from Tel Aviv, Israel.

of Göttingen, Germany. The network of these two telescopes will increase the number of measurements, enhancing the expected number of planets. Dozens of transiting extrasolar planets are expected to be found over the three year observation campaign.

\section{References}

Alonso, R. et al., ApJ 613L, 153

Butler, B. et al., ApJ 617, 580B

Charbonneau, D. et al., ApJ 529L, 45

COROT, http://sci.esa.int/home/corot/index.cfm

Kepler, http://www.kepler.arc.nasa.gov

Marcy, G. \& Butler, R .P., ApJ 464, L147

Mayor, M. \& Queloz, D., Nature 378, 355

McArthur, B. et al., ApJ 614L, 81M

MONET, http://monet.uni-goettingen.de/cgi-bin/WebObjects/MonetPortal

Rivera, E. et al., ApJ 634, 625

Santos, N. et al., A\&SA 426L, 19S

Planets Encyclopedia, http://www.obspm.fr/planetes

Udalski, A. et al., Acta. Astron. 52, 115 